\title{
Low energy light-by-light scattering in heavy-ion UPC, a new possibility
}

\section{Mariola Kłusek-Gawenda ${ }^{* \dagger}$}

Institute of Nuclear Physics Polish Academy of Sciences, PL-31342 Krakow, Poland

E-mail: mariola.klusekeifj.edu.pl

This work will be focused on the analysis of the light-by-light scattering in the mass range $W_{\gamma \gamma}<$ $5 \mathrm{GeV}$. Here contribution from fermionic boxes, resonance scattering $\left(\eta, \eta^{\prime}(958), \eta_{c}(1 S), \eta_{c}(2 S)\right.$, $\left.\chi_{c 0}(1 P) \rightarrow \gamma \gamma\right)$ and pionic background will be compared. The total cross section for the signal and the background at midrapidity $|\eta|<0.9$, and in the forward rapidity range $2.0<\eta<4.5$ will be presented. Also, the differential cross section will be considered for the next run at the LHC.

XXVII International Workshop on Deep-Inelastic Scattering and Related Subjects - DIS2019 8-12 April, 2019

Torino, Italy

* Speaker.

${ }^{\dagger}$ This research was supported in part by the Polish National Science Center grant DEC-2014/15/B/ST2/02528 


\section{Introduction}

Physics of the ultra-peripheral collisions (UPC) of heavy ions gives a nice opportunity to study electromagnetic processes. Due to the very strong electromagnetic field of colliding nuclei, reactions relate to photon collisions can be studied. One can consider $\gamma \gamma$ fusion and photoproduction (Pomeron and/or Reggeon exchange) as a sub-process of heavy ion UPC. This proceedings will pertain to light-by-light scattering. Di-photon processes have long been studied at $e^{+} e^{-}$collider. This tool allows to test a QED theory and a lot of aspects of meson spectroscopy. The first theory concerning the possibility of the light-by-light scattering was proposed more than 80 years ago i.a. by W. Heisenberg and his students : H. Euler and B. Kockel $[1,2]$ or by A. Akhieser, L. Landau and I. Pomeranchuk [3].

The first evidence of di-photon measurements in ultra-peripheral heavy-ion collisions have been reported by the ATLAS and CMS Collaborations [4, 5]. These data are, however, restricted to photon-photon invariant masses $\mathrm{W}_{\gamma \gamma}>5$ and $6 \mathrm{GeV}$ for the CMS and ATLAS analysis, respectively. ATLAS measured a fiducial cross section of $\sigma=70 \pm 24$ (stat.) \pm 17 (syst.) nb [4] and $\sigma=78 \pm 13$ (stat.) \pm 7 (syst.) \pm 3 (lumi.) nb [6] and our theoretical calculations (including experimental acceptance) gave $51 \pm 0.02 \mathrm{nb}$ [7]. In comparison with ATLAS result, the CMS fiducial light-by-light scattering cross section equal to $\sigma=120 \pm 46$ (stat.) \pm 28 (syst.) \pm 4 (theo.) $\mathrm{nb}$. Our differential and total cross section $(\sigma=103 \pm 0.034 \mathrm{nb})$ is in a good agreement with the CMS result.

(a)

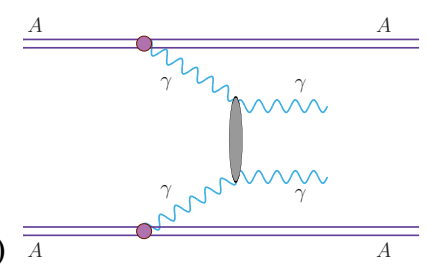

(b)

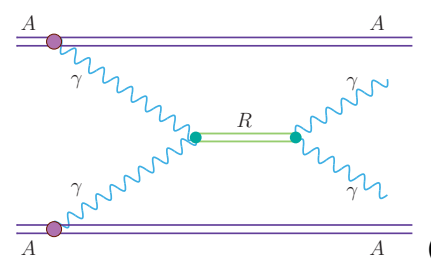

(c)

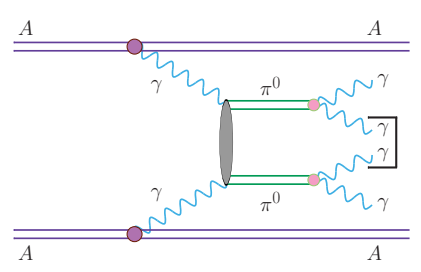

Figure 1: Diagrams for nuclear $A A \rightarrow A A \gamma \gamma$ mechanism. Different subprocesses can be signalized: (a) Standard Model box (b) resonances (c) pionic background.

A current analysis concerns the production of di-photon pairs originating from boxes (see Fig. 1(a)), resonances which decay into $\gamma \gamma$ state (see Fig. 1(b)) and pionic background (see Fig. 1(c)).

\section{Theoretical approach for signal and background}

The leading order of elementary cross section for $\gamma \gamma \rightarrow \gamma \gamma$ process is well-know and one can use an available to the general public Mathematica package: FormCalc [8]. So-called fermionic box means scattering via quarks and leptons. Also, $W^{+} W^{-}$boson loop can be included but bosonic contribution appears only at twice mass of $W$ boson. The role of mesons exchanges in the $\gamma \gamma \rightarrow \gamma \gamma$ process was comprehensively studied in Ref. [9]. We take into account only pseudoscalar and scalar mesons: $\eta, \eta^{\prime}(958), \eta_{c}(1 S), \eta_{c}(2 S)$ and $\chi_{c 0}(1 P)$. The amplitude and cross section for the $\gamma \gamma$ production through the $s$-channel exchange of a pseudoscalar/scalar mesons is determined using notation as in Ref. [10]. Their masses $m_{R}$, total widths $\Gamma_{R}$ and branching ratios $\operatorname{Br}(R \rightarrow \gamma \gamma)$ are taken from PDG [11]. 
In addition, also background from the $\gamma \gamma \rightarrow \pi^{0}(\rightarrow \gamma \gamma) \pi^{0}(\rightarrow \gamma \gamma)$ process is considered. In Ref.[10] we constructed a multi-component model which described e.g. the Belle [12] and Crystal Ball [13] data for $\gamma \gamma \rightarrow \pi^{0} \pi^{0}$. In [10] both $\gamma \gamma \rightarrow \pi^{+} \pi^{-}$and $\gamma \gamma \rightarrow \pi^{0} \pi^{0}$ reactions were considered within a multi-component model. There, for the first time, both the total cross section and angular distributions and significance of nine resonances, $\gamma \gamma \rightarrow \pi^{+} \pi^{-} \rightarrow \rho^{ \pm} \rightarrow \pi^{0} \pi^{0}$ continuum, Brodsky-Lepage and handbag mechanisms in these processes was studied. If only two photons from different neutral pions are measured at a given experimental range of rapidities and transverse momenta such an event could be wrongly identified as $\gamma \gamma \rightarrow \gamma \gamma$ scattering if no extra cuts are imposed to reduce or eliminate such a background.

The nuclear cross section is calculated with the help of equivalent photon approximation (EPA) $[14,15,16]$. This theory uses the concept of photon fluxes which depend on energy of photon $\left(\omega_{i}\right)$ and impact parameter $\left(\mathbf{b}_{i}\right)$. Here we consider only ultra-peripheral collisions that is impact parameter $(b)$ determining the distance between the medium of colliding nuclei is bigger than the sum of the radii. Fig. 2(a) reflects quantities which are used in the impact parameter space. The end of $\mathbf{b}_{i}$ vectors set a position where the pair of particles is produced. Here we mean just two-photon production. A schematic diagram illustrating the process of heavy ion collision that is associated with the production of some final state $X$ is presented in Fig.2(b). It is often used rather photon number $\left(n\left(\omega_{i}\right)\right)$ instead of photon flux $\left(N\left(\omega_{i}, \mathbf{b}_{i}\right)\right)$ (where $i=1,2$ numbers nuclei) but we think that usage of EPA in the impact parameter space is more accurate. To calculate nuclear cross section for $A A \rightarrow A A X$ process one needs to know the probability that the first nucleus emits a photon with the energy $\omega_{1}$ and, simultaneously, the second nucleus emits a photon with the energy $\omega_{2}$. Next, they collide with each other to produce some final state $X$ (here it is $\gamma \gamma$ or $\pi^{0} \pi^{0}$ state).

(a)

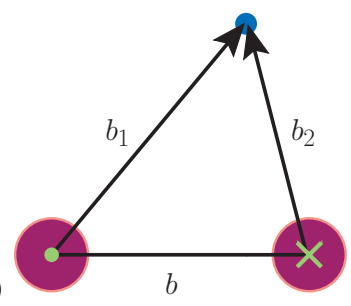

(b)

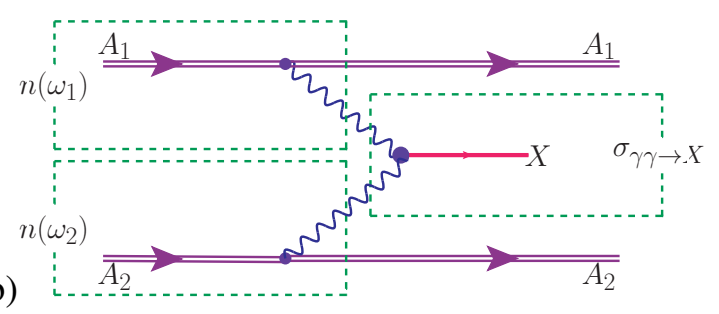

Figure 2: (a) A view perpendicular to the direction of two ion motion. (b) Feynman diagram for AA $\rightarrow \mathrm{AAX}$ process.

In our approach, we use EPA in the impact parameter space. The cross section takes the form:

$$
\begin{aligned}
\sigma_{A_{1} A_{2} \rightarrow A_{1} A_{2} X_{1} X_{2}}\left(\sqrt{s_{A_{1} A_{2}}}\right) & =\int \sigma_{\gamma \gamma \rightarrow X_{1} X_{2}}\left(W_{\gamma \gamma}\right) N\left(\omega_{1}, \mathbf{b}_{\mathbf{1}}\right) N\left(\omega_{2}, \mathbf{b}_{\mathbf{2}}\right) S_{a b s}^{2}(\mathbf{b}) \\
& \times \mathrm{d}^{2} b \mathrm{~d} \bar{b}_{x} \mathrm{~d} \bar{b}_{y} \frac{W_{\gamma \gamma}}{2} \mathrm{~d} W_{\gamma \gamma} \mathrm{d} Y_{X_{1} X_{2}},
\end{aligned}
$$

where $X_{1} X_{2}$ is a pair of produced particles, $\bar{b}_{x}$ and $\bar{b}_{y}$ are components of $\mathbf{b}_{i}$ vector, the energy of produced particles depends on energy of photons as follows: $W_{X_{1} X_{2}}=2 \sqrt{\omega_{1} \omega_{2}}$ and rapidity of outgoing system is expressed through rapidity of singles particles: $Y_{X_{1} X_{2}}=\frac{1}{2}\left(y_{X_{1}}+y_{X_{2}}\right)$. Absorption factor $S_{a b s}(\mathbf{b})$ assures UPC which means that the nuclei do not undergo nuclear breakup. Very often we extend Eq. (2.1) by additional dimension. This allows to make a more accurate prediction or description of experimental data. 


\section{Nuclear predictions}

Light-by-light scattering was realized experimentally only recently $[4,6,5]$. About one year before the first experimental results which were obtained by the ATLAS group, we have studied light-by-light scattering via fermion loop and with the help of non-perturbative mechanism of photons fluctuation into light vector mesons (VDM-Regge model) [7]. We have considered only ultra-peripheral lead-lead collisions. For ions of charges $Z_{1}, Z_{2}$, the cross section is enhanced by $Z_{1}^{2} Z_{2}^{2}$ factor compared to proton-proton collisions, at least at low di-photon invariant masses equal to di-photon collision energies, where the initial photons are quasi real with extremely low virtualities. But on the other hand, a significant part of cross section is cut by absorption factor (Eq. (2.1)) which ensures ultra-peripheral character of the process.

The ALICE detector facilitates a measurement of outgoing photons at midrapidity region $\left|\eta_{\gamma}\right|<0.9$ [17]. Photons with transverse energy smaller than $200 \mathrm{MeV}$ cannot be detected. Calculations for ALICE (semi)acceptance will be compared with the results for more forward rapidity region: $2<\eta<4.5$, what corresponds to LHCb fiducial region [18]. Here we assume that any photon with $p_{t, \gamma}>200 \mathrm{MeV}$ will be measured.

Table 1: Total nuclear cross section in nb, $\sqrt{s_{N N}}=5.02 \mathrm{TeV}$.

\begin{tabular}{l|rr|rr}
\hline Energy & \multicolumn{2}{|c}{$W_{\gamma \gamma}=(0-2) \mathrm{GeV}$} & \multicolumn{2}{c}{$W_{\gamma \gamma}>2 \mathrm{GeV}$} \\
\hline Fiducial region & ALICE & LHCb & ALICE & LHCb \\
\hline boxes & 4890 & 3818 & 146 & 79 \\
$\pi^{0} \pi^{0}$ background & 135300 & 40866 & 46 & 24 \\
$\eta$ & 722573 & 568499 & & \\
$\eta^{\prime}(958)$ & 54241 & 40482 & & \\
$\eta_{c}(1 S)$ & & & 9 & 5 \\
$\chi_{c 0}(1 P)$ & & & 4 & 2 \\
$\eta_{c}(2 S)$ & & & 2 & 1 \\
\hline
\end{tabular}

In Table 1 one can find values of the total nuclear cross section for fermionic boxes, pionic background and five types of mesons. Cross section is given in two ranges of the di-photon invariant masses. The first range is from 0 to $2 \mathrm{GeV}$, the second range is for values $W_{\gamma \gamma}$ larger than 2 $\mathrm{GeV}$. We take $W_{\gamma \gamma}^{\max }=50 \mathrm{GeV}$ for fermionic boxes, $W_{\gamma \gamma}^{\max }=5 \mathrm{GeV}$ for the $\pi^{0} \pi^{0}$ background (it is negligible above $\left.W_{\gamma \gamma}=5 \mathrm{GeV}\right)$ and $W_{\gamma \gamma} \in\left(m_{R}-1 \mathrm{GeV}, m_{R}+1 \mathrm{GeV}\right)$ for resonances. Here a cut on pseudorapidity and energy or transverse momentum of photons is included. The largest cross section is obtained for the $\gamma \gamma \rightarrow \eta \rightarrow \gamma \gamma$ resonance scattering. Additionally, in the range of diphoton invariant mass $M_{\gamma \gamma}>2 \mathrm{GeV}$, comparison of cross sections for fermionic boxes and pionic background clearly shows almost fourfold dominance of boxes over the unwanted background.

The two final state photons of $\gamma \gamma \rightarrow \gamma \gamma$ scattering are of equal transverse momenta and are back-to-back in azimuthal angle. The correlation in transverse momentum will be smeared out by the experimental resolution in the measurement of the two photons. The energy resolution for LHCb is parametrized as [19]: $\frac{\sigma_{E_{\gamma}}}{E_{\gamma}}=\frac{0.085}{\sqrt{E_{\gamma}}}+\frac{0.003}{E_{\gamma}}+0.008$, where $E_{\gamma}$ is the photon energy in GeV and ALICE parametrization takes the constant form $\sigma_{E_{\gamma}} / E_{\gamma} \sim 1.3 \%$ [20]. Inclusion of the Gaussian distribution to simulate experimental energy resolution causes only a little smearing of the resonant 
signals. Then the transverse momenta of each of the photons takes the form: $p_{i, t}=p_{t}+\left(\frac{p_{t}}{E_{i}}\right) \delta E_{i}$. It seems to worth mentioning that the peak corresponding to a resonance very strongly depends on the number of bins. The maximum value of the differential cross section emerges exactly at $m_{R}$.

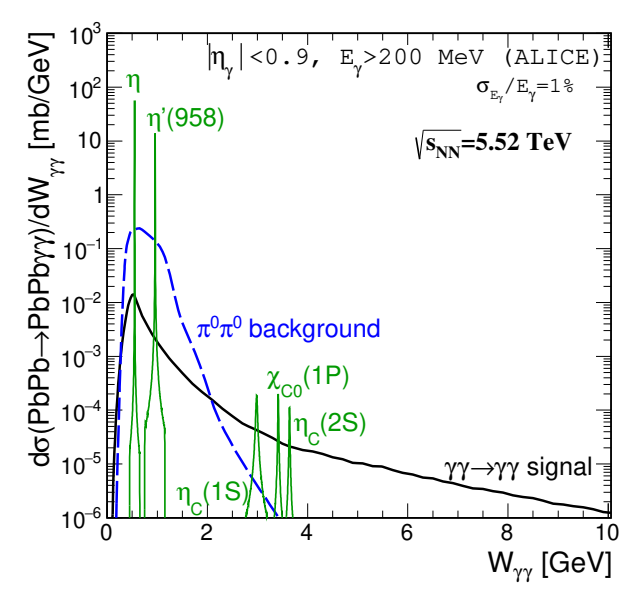

(a)

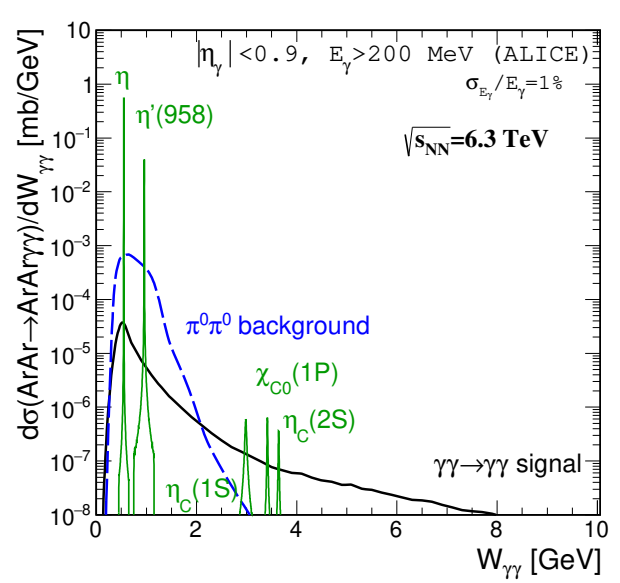

(b)

Figure 3: Differential cross section as a function of $W_{\gamma \gamma}=M_{\gamma \gamma}$ for (a) $\mathrm{PbPb} \rightarrow \mathrm{PbPb} \gamma \gamma$ and (b) $\mathrm{ArAr} \rightarrow \operatorname{ArAr} \gamma \gamma$. The collision energy at the center-of-mass of the heavy ion collision is $5.52 \mathrm{TeV}$ and 6.3 TeV for lead-lead and argon-argon respectively.

Fig. 3 corresponds to the predictions for the next run at the LHC. The energy (per nucleon) of heavy-ion collision was calculated as follows: $\sqrt{s_{N N}}=\sqrt{\left(Z_{1} Z_{2}\right) /\left(A_{1} A_{2}\right)} \times \sqrt{s_{p p}}$ for the energy in the center-of-mass of proton-proton collision $\sqrt{s_{p p}}=14 \mathrm{TeV}$. Then predicted values for ${ }^{208} \mathrm{~Pb}^{82+}$ ${ }^{208} \mathrm{~Pb}^{82+}$ collision is $\sqrt{s_{N N}}=5.52 \mathrm{TeV}$ (Fig. 3(a)) and for ${ }^{40} \mathrm{Ar}^{18+}{ }_{-}^{40} \mathrm{Ar}^{18+}$ it is $\sqrt{s_{N N}}=6.3 \mathrm{TeV}$ (Fig. 3(b)). At lower energies ( $W_{\gamma \gamma}<4 \mathrm{GeV}$ ) meson resonances may play important role in addition to the Standard Model box diagrams or proposed pionic background. The inclusion of energy resolution has a significance mainly at $\gamma \gamma \rightarrow \eta, \eta^{\prime} \rightarrow \gamma \gamma$ resonance scattering and this contribution will be measured with good statistics. However, the resonance signal is modified including experimental energy resolution and the $\eta$ and $\eta^{\prime}(958)$ peaks are about one order of magnitude smaller than without experimental resolution but the total cross section is of course still the same. These figures suggest that one could be able to measure the $\gamma \gamma \rightarrow \gamma \gamma$ scattering above $W_{\gamma \gamma}>2 \mathrm{GeV}$. The acoplanarity requirement of 0.01 reduces the background contribution by a factor of 5 in the full $W_{\gamma \gamma}$ region. Comparing Fig. 3 (a) with (b) one can observe that the relevant distribution varies more than two orders of magnitude. In the case of argon-argon collisions, although the collision energy is larger, the predicted cross section is smaller. This is caused by the fourth power of the charge number of the nucleus in the cross section. The photon flux depends on $Z_{A}^{2}$ so the cross section is multiplied by $Z_{A}^{4}$. Thus the total cross section for lead-lead collision is more than two orders of magnitude larger than for the argon-argon collision case.

\section{Conclusion}

The ultra-peripheral heavy ion collisions give a possibility to measure the $\gamma \gamma \rightarrow \gamma \gamma$ scattering. So far the ATLAS and CMS collaborations measured the light-by-light scattering for di-photon collisions energies $W_{\gamma \gamma}>6 \mathrm{GeV}$ (ATLAS) and $W_{\gamma \gamma}>5 \mathrm{GeV}$ (CMS). Calculated by us the Standard 
Model predictions are roughly consistent with the experimental data. Our results include realistic photon fluxes that are a Fourier transform of the charge distribution in the nucleus. We have proposed several additional mechanisms which contribute to two-photon state. Each of them plays an important role and different ranges of di-photon invariant mass or transverse momentum of a single photon. Next, we have studied background from two-pion decay into pairs of photons. Here the condition that only two out of four photons in the final state are detected. This contribution could be wrongly interpreted as an enhanced $\gamma \gamma \rightarrow \gamma \gamma$ scattering at low energies $\left(M_{\gamma \gamma}<2 \mathrm{GeV}\right)$. The signal to background ratio will be improved by including extra cut i.e. on scalar/vector asymmetry [21], transverse momentum of the $\gamma \gamma$ pair [22] or acoplanarity [23]. The $\gamma \gamma$ scattering through pseudoscalar and scalar mesons $\left(\eta, \eta^{\prime}(958), \eta_{c}(1 S), \eta_{c}(2 S), \chi_{c 0}(1 P)\right)$ have been studied too. The $\gamma \gamma \rightarrow \eta, \eta^{\prime} \rightarrow \gamma \gamma$ resonance scattering could be measured with good statistics. Comparing contributions for lead-lead and argon-argon collisions, one can deduce that collision of lighter nuclei is less favorable. However, we can hope the luminosity in the run with Ar-Ar collision will be higher.

\section{References}

[1] H. Euler and B. Kockel, Naturwiss. 23, 246 (1935)

[2] W. Heisenberg and H. Euler Zeit. f. Phys. 98, 714 (1936)

[3] A. Akhieser, L. Landau and I. Pomeranchuk, Nature 138, 206 (1936)

[4] ATLAS Collaboration (M. Aaboud et al.), Nature Phys. 13, 852 (2017)

[5] CMS Collaboration (A. M. Sirunyan et al.), arXiv:1810.04602 [hep-ex]

[6] ATLAS Collaboration (G. Aad et al.), Phys. Rev. Lett. 123 (2019) 052001

[7] M. Kłusek-Gawenda, P. Lebiedowicz and A. Szczurek, Phys. Rev. C93, 044907 (2016)

[8] T. Hahn and M. Perez-Victoria, Comput. Phys. Commun. 118, 153 (1999)

[9] P. Lebiedowicz and A. Szczurek, Phys. Lett. B772, 330 (2017)

[10] M. Kłusek-Gawenda and A. Szczurek, Phys. Rev. C87, 054908 (2013)

[11] Particle Data Group (C. Patrignani et al.), Chin. Phys. C40, 100001 (2016)

[12] Belle Collaboration (S. Uehara et al.), Phys. Rev. D79, 052009 (2009)

[13] Crystal Ball Collaboration (H. Marsiske et al.), Phys. Rev. D41, 3324 (1990)

[14] E. Fermi, Nuovo Cimento 2, 143 (1925)

[15] C. F. Weizsäcker, Z. Phys. 88, 612 (1934)

[16] E. J. Williams, Phys. Rev. 45, 729 (1934)

[17] ALICE Collaboration (B. B. Abelev et al.), Int. J. Mod. Phys. A29, 1430044 (2014)

[18] LHCb Collaboration (M. Clemencic et al.), J. Phys. Conf. Ser. 331, 032023 (2011)

[19] C. Abellan Beteta et al., JINST 7 (2012) P08020

[20] ALICE Collaboration (S. Acharya et al.), Eur. Phys. J. C78 (2018) 263

[21] M. Kłusek-Gawenda, R. McNulty, R. Schicker and A. Szczurek, Phys. Rev. D99 (2019) 093013

[22] M. Kłusek-Gawenda, arXiv:1809.03823 [hep-ph]

[23] Z. Citron et al., arXiv: 1812.06772 [hep-ph] 